\title{
Operative and hormonal management of recurrent catamenial pneumothorax: A case report
}

\author{
Sivaraman K lyer BS, Jessica Clay Ehrig MD, Marcos Sosa MD, Jennifer Phy DO
}

\begin{abstract}
Catamenial pneumothorax refers to the recurrent collapse of a lung in conjunction with the menstrual cycle. It is a common manifestation of thoracic endometriosis syndrome, a rare condition involving extra-pelvic endometrial lesions. We describe a case of catamenial pneumothorax that has not recurred after treatment with hysterectomy and bilateral salpingooophorectomy. A 37-year-old woman with abdominal endometriosis presented with five episodes of right pneumothorax, all of which coincided with the patient's menstrual cycle. The diagnosis of catamenial pneumothorax was made, and the patient was referred for hysterectomy and bilateral salpingo-oophorectomy. Combined transdermal estrogen/progesterone treatment was also initiated to manage her symptoms of hot flashes, vaginal dryness, and dyspareunia. Since treatment, the patient has not had recurrent pneumothorax. Due to the rarity and acuity of catamenial pneumothoraces, management options are challenging. Recommendations on the duration of combined estrogen/progesterone treatment following bilateral oophorectomy are needed. A multidisciplinary team approach consisting of pulmonologists, thoracic surgeons, and gynecologists is essential for accurate diagnosis, optimal treatment, and successful outcomes in this rare but serious condition.
\end{abstract}

Keywords: Catamenial pneumothorax, endometriosis/therapy, thoracic diseases, menstrual cycle

\section{INTRODUCTION}

Endometriosis is defined as the presence of endometrial tissue outside of the uterine cavity. ${ }^{1}$ It usually occurs within the pelvis, with the most common sites of implantation on the fallopian tubes, ovaries, and pelvic peritoneum. Treatment of common lesions includes administration of pharmacologic agents to suppress endometriosis or surgical ablation. ${ }^{1}$ However, endometrial lesions can occur in atypical locations, including the lower genitourinary tracts, gastrointestinal

Corresponding author: Jennifer Phy

Contact Information: Jennifer.phy@ttuhsc.edu

DOI: 10.12746/swrccc.v6i26.494 tract, skin, nervous system, and the thoracic cavity. ${ }^{1,2}$ The most common location of extra-pelvic endometrial lesions is in the thoracic cavity (see Figures 1 and 2 ), collectively referred to as thoracic endometriosis. ${ }^{3,4}$ The clinical manifestations of thoracic endometriosis include catamenial pneumothorax, catamenial hemothorax, catamenial hemoptysis, and lung nodules. ${ }^{5}$ The term "catamenial" refers to the temporal relationship of these events with menses. Although thoracic endometriosis is itself a rare phenomenon and its prevalence is uncertain, catamenial pneumothorax is its most common clinical manifestation accounting for $3-6 \%$ of cases of women suffering from spontaneous pneumothorax. ${ }^{6}$

We describe a patient with both abdominal and thoracic endometriosis who presented to our practice with five episodes of catamenial pneumothorax. 


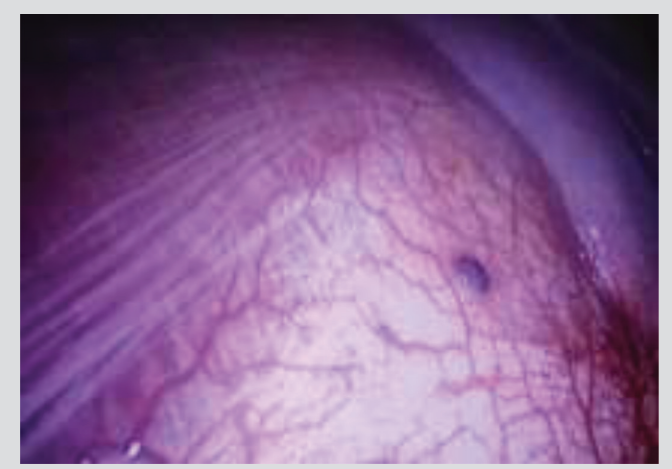

Figure 1. Pleural lesion of endometriosis (dark purple lesion).

Figure 1 and 2 were downloaded from the OPENi(beta) image resource collection at Texas Tech University Health Sciences Center in Lubbock, Texas. Accessed $6 / 10 / 2018$.

\section{CASE}

A 37-year-old primiparous Caucasian woman presented with pneumothorax in January 2009. Her past medical history was significant for autoimmune hepatitis and symptomatic abdominal endometriosis status post laparoscopy. Her pneumothorax was successfully treated and she was discharged. She was re-hospitalized with two subsequent pneumothoraces in February and March 2009 and underwent chemical talc pleurodesis. In May 2009, she returned with a fourth pneumothorax and was referred to cardiothoracic surgery for management. At this time, a talc and polyglactin mesh were placed, and right-sided diaphragmatic fenestrations were noted without evidence of endometrial implants.

The patient returned a fifth time with a pneumothorax. Physical examination revealed diminished breath sounds in the right lung base in an area that corresponded to a $4 \mathrm{~cm}$ area of scaring from her previous surgery. Pelvic examination and transvaginal ultrasound showed a retroverted uterus deviating to the patient's left with adnexa tender to palpation. No obvious adnexal masses or ovarian cysts were palpated. During this hospitalization the patient and the clinical team noted that the pneumothoraces corresponded to her menstrual cycle, and a working diagnosis of catamenial pneumothorax was adopted. After counseling, the patient chose to have a total abdominal hysterectomy and bilateral salpingo-oophorectomy. Because the patient also had symptomatic abdominal endometrial lesions, she was also placed on leuprolide acetate (depot Lupron) $3.75 \mathrm{mg}$ injection and norethindrone (Aygestin) $5 \mathrm{mg}$ "addback" therapy daily as needed for sleeplessness, headaches, hot flashes, dyspareunia, and vaginal dryness. Since her surgery, the patient has not had a recurrent pneumothorax. With the combined hormone therapy, her hot flashes and vaginal dryness have subsided.

\section{Discussion}

Although catamenial pneumothorax is not a commonly considered diagnosis, it should be included in the differential diagnosis of a woman of childbearing age who presents with pneumothorax, pneumothorax requiring surgery, or recurrent pneumothorax. The incidence rates of catamenial pneumothorax are uncertain, but one retrospective study from a French tertiary referral center reported a rate as high as $24.6 \%$ in 114 women of childbearing age referred

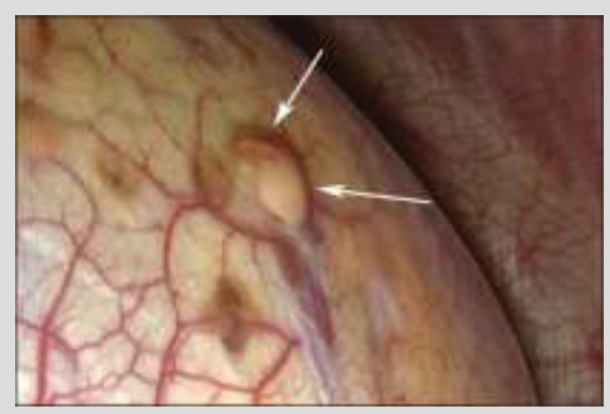

Figure 2. Pleural lesion of endometriosis (raised lesion at arrows).

Figure 1 and 2 were downloaded from the OPENi(beta) image resource collection at Texas Tech University Health Sciences Center in Lubbock, Texas. Accessed 6/10/2018. 
for video-assisted thoracic surgery for spontaneous pneumothorax. ${ }^{7}$ The study also demonstrated that a majority, but not all, of patients with catamenial pneumothorax may have endometriotic implants within the thorax. ${ }^{7}$

The key element to diagnosing catamenial pneumothorax is uncovering the temporal relationship between the pneumothorax and the onset of menses. In most patients, the clinical symptoms of chest pain and dyspnea occur within 48 hours of menstruation onset. ${ }^{8}$ A meta-analysis of 44 thoracic endometriosis and catamenial pneumothorax case reports published between January 2001 and July 2007 yielded 110 cases. Of these cases of thoracic endometriosis, $72 \%$ presented initially with catamenial pneumothorax, and $14 \%$ presented initially with hemoptysis. ${ }^{9}$ The majority of catamenial pneumothoraces (up to $63 \%$ ) occur on the right side. ${ }^{10}$ Imaging studies, such as computed tomography or magnetic resonance imaging, can support a clinical suspicion of catamenial pneumothorax, but the key features to support the diagnosis are the relationship with menses, cyclical changes in imaging studies, and posterosuperior diaphragmatic lesions. ${ }^{4}$ Thoracoscopy is the most widely used diagnostic tool since it is both diagnostic and therapeutic. ${ }^{4}$

Therapy should be focused on immediate expansion of the lung. While pleurodesis is an effective treatment, it should be avoided in young women with catamenial pneumothorax until video-assisted thoracoscopic surgery (VATS) can be used to confirm the diagnosis since pleurodesis can mask small lesions. ${ }^{11}$ Once the diagnosis of catamenial pneumothorax has been established, VATS can be used to excise endometrial lesions where possible. ${ }^{6}$ Medical treatment of catamenial pneumothorax with $\mathrm{GnRH}$ analogues before surgery can suppress recurrences; however, it should be noted that due to the rarity of this condition there are no randomized controlled trials on the treatment of catamenial pneumothorax. ${ }^{12}$ In addition, one study has shown that catamenial pneumothorax can potentially recur if hormone replacement therapy is initiated after surgery. ${ }^{7}$ Our patient benefited from hysterectomy with bilateral salpingo-oophorectomy, which has been cited in the literature as a potential definitive treatment for catamenial pneumothorax ${ }^{7}$
While this is not an option for women who want to preserve their fertility, we advocate for this method for the treatment of both catamenial pneumothorax and abdominal endometrial lesions when indicated.

The key to properly treating female patients with recurrent catamenial pneumothorax is taking a careful history. After the diagnosis is made, it takes gynecologists, pulmonologists, and thoracic surgeons working as a team to recognize, diagnosis, treat, and educate patients with catamenial pneumothorax. We advocate for a combined treatment approach using gynecologic and thoracic surgical techniques along with hormonal treatment to minimize symptoms and to reduce risk of recurrence in this rare but serious condition.

Article citation: Iyer SK, Jessica Clay Ehrig JC, Marcos Sosa M, Phy J. Operative and hormonal management of recurrent catamenial pneumothorax: a case report. The Southwest Respiratory and Critical Care Chronicles 2018;6(26):22-25

From: Texas Tech University Health Sciences Center, Department of Obstetrics and Gynecology, Division of Reproductive Endocrinology and Infertility, Lubbock, TX (SKI, JP); University of Colorado, Department of Obstetrics and Gynecology, Division of Maternal Fetal Medicine, Aurora, CO (JCE); Little River Healthcare, Department of Women's Health, Killeen, TX (MS)

Submitted: $4 / 7 / 2018$

Accepted: $7 / 15 / 2018$

Reviewers: Chok Limsuwat MD, Anoop Nambiar MD Conflicts of interest: none

This work is licensed under a Creative Commons Attribution-ShareAlike 4.0 International License

\section{REFERENCES}

1. Olive DL, Schwartz LB. Endometriosis. N Engl J Med 1993; 328(24):1759-1769.

2. Lee HJ, Park YM, Jee BC, et al. Various anatomic locations of surgically proven endometriosis: A single-center experience. Obstetrics \& Gynecology Science 2015;58(1):53-58.

3. Bagan P, Berna P, Assouad J, et al. Value of cancer antigen 125 for diagnosis of pleural endometriosis in females with recurrent pneumothorax. European Respiratory $J$ 2008; 31(1):140-142. 
4. Rousset P, Rousset-Jablonski C, Alifano M, et al. Thoracic endometriosis syndrome: CT and MRI features. Clinical Radiology 2014;69(3):323-330.

5. Azizad-Pinto P, Clarke D. Thoracic endometriosis syndrome: case report and review of the lite rature. The Permanente Journal 2014;18(3):61-65.

6. Visouli AN, Zarogoulidis K, Kougioumtzi I, et al. Catamenial pneumothorax. J Thoracic Disease 2014;6(Suppl 4): S448-S460.

7. Alifano M, Jablonski C, Kadiri H, et al. Catamenial and noncatamenial, endometriosis-related or nonendometriosis-related pneumothorax referred for surgery. American J Respiratory Critical Care Medicine 2007;176(10):1048-1053.

8. Joseph J, Sahn SA. Thoracic endometriosis syndrome: new observations from an analysis of 110 cases. The American $J$ Medicine 1996;100(2):164-170.
9. Channabasavaiah AD, Joseph JV. Thoracic endometriosis: revisiting the association between clinical presentation and thoracic pathology based on thoracoscopic findings in 110 patients. Medicine 2010;89(3).

10. Legras A, Mansuet-Lupo A, Rousset-Jablonski C, et al. pneumothorax in women of child-bearing age: an update classification based on clinical and pathologic findings. Chest 2014;145(2):354-360.

11. Korom S, Canyurt H, Missbach A, et al. Catamenial pneumothorax revisited: Clinical approach and systematic review of the literature. The J Thoracic and Cardiovascular Surgery 2004;128(4):502-508.

12. Attaran S, Bille A, Karenovics W, et al. Videothoracoscopic repair of diaphragm and pleurectomy/abrasion in patients with catamenial pneumothorax: a 9-year experience. Chest 2013;143(4):1066-1069. 